\title{
Extracorporeal membrane oxygenation in cardiac arrest
}

Boon Kiat Kenneth Tan, MBBS, MCEM

\begin{abstract}
The survival of cardiac arrest patients is dependent on the efficient and timely application of the chain of survival. This includes early identification of cardiac arrest and activation of the emergency response system, high-quality cardiopulmonary resuscitation (CPR), rapid defibrillation, basic and advanced medical services, and advanced life support and post-cardiac arrest care. However, some patients may be refractory to these measures. Extracorporeal membrane oxygenation CPR (ECPR), otherwise known as extracorporeal life support, offers an alternative to such refractory cardiac arrest cases. As hospitals have limited resources, it is important to devise effective patient selection methods, and improve our understanding and experience of ECPR to ensure the best outcome. It is also vital to understand that ECPR constitutes only one component of post-cardiac arrest care, which includes other aspects such as therapeutic hypothermia and early perfusion for best patient outcome.
\end{abstract}

Keywords: cardiac arrest, ECPR, patient selection, survival

\section{INTRODUCTION}

The survival of cardiac arrest patients is dependent on the chain of survival being carried out in an efficient and timely manner, starting with early identification of cardiac arrest and activation of the emergency response system, followed by high-quality cardiopulmonary resuscitation (CPR), rapid defibrillation, basic and advanced medical services, and advanced life support and post-cardiac arrest care. However, some patients are refractory to such measures. Extracorporeal membrane oxygenation (ECMO) CPR (ECPR), otherwise known as extracorporeal life support (ECLS), offers an alternative in such refractory cardiac arrest cases.

\section{TYPES OF ECMO}

ECMO can be divided into two main configurations: venoarterial (VA) and venovenous (VV). In the VA configuration, ECMO catheters are inserted through a large vein (femoral vein or superior vena cava) and a large artery (femoral artery or carotid artery); this is mainly used for patients in cardiogenic shock or on ECPR/ECLS. In the VV configuration, which is usually deployed during respiratory failure that is refractory to conventional treatment, both of the catheters are inserted into large veins. During refractory cardiac arrest, ECPR/ECLS can be deployed. The ECMO machine is implanted after the ECMO catheters are inserted via ultrasonography guidance using either the Seldinger or surgical cutdown technique during ongoing CPR. The patient must be deemed suitable for ECPR and have no contraindications to ECMO implantation.

\section{EVIDENCE FOR ECPR \\ In children}

The use of ECMO is more established in children than in adults. Brown and Dalton ${ }^{(1)}$ discussed various studies in which ECPR was performed in highly supervised areas, such as the intensive care unit or operating theatre, in patients with congenital or acquired cardiac diseases. The authors also highlighted a small group of studies on out-of-hospital cardiac arrest (OHCA) that were mostly related to hypothermic incidents such as submersion. (1) One such study was conducted by Wollenek et al, ${ }^{(2)}$ who reported that ten out of the 12 children who suffered OHCA due to hypothermia survived, although all but one of the survivors had some form of neurological deficit. Chan et al ${ }^{(3)}$ postulated that children with more complex congenital heart diseases (e.g. single ventricles, severe metabolic acidosis) had worse outcomes.

\section{In adults}

On 1 January 2015, the Extracorporeal Life Support Organization (ELSO) released the International ECLS registry report, which was based on its experience with 1,657 ECPR patients. A total of 639 (39\%) patients survived initial ECPR; of these, 471 (74\%) patients survived till discharge. ${ }^{(4)}$ Cardarelli et al ${ }^{(5)}$ performed a meta-analysis of a series of observational studies on ECPR involving 135 adult patients. Although the neurological sequelae and complications were not well documented, the study showed some important results: the overall survival to hospital discharge was $40 \%$ and the most common cause of cardiac arrest was acute myocardial infarction. More importantly, those in the younger age group (17-41 years) had a higher survival rate compared to those in the 41-56 years age group (odds ratio [OR] 2.9; 95\% confidence interval $[\mathrm{Cl}] 1.6-8.2)$ and $>67$ years age group (OR $3.4 ; 95 \% \mathrm{Cl} 1.2-9.7)$. Cardarelli et al also showed that patients who had CPR performed for more than 30 minutes were more likely to die than those who received less than 30 minutes of CPR (OR 1.9; 95\% Cl 0.9-4.2). ${ }^{(5)}$ The data suggests that ECPR may have better outcomes in younger patients who receive a shorter duration of CPR with early implantation of ECMO.

Sakamoto et $\mathrm{al}^{(6)}$ studied the difference in neurological outcomes between ECPR and conventional CPR (CCPR) in OHCA 
patients with ventricular fibrillation (VF) or pulseless ventricular tachycardia (VT) at one month and six months after cardiac arrest. Neurological outcomes were defined by the Cerebral Performance Category (CPC) and results were obtained using an intention-to-treat analysis. At one month, 13.7\% (32/234) of the ECPR group were CPC 1-2 compared to $1.9 \%$ (3/159) of the CCPR group ( $p<0.0001)$. At six months, $12.4 \%$ of the ECPRgroup were CPC 1-2 compared to $3.1 \%$ of the CCPR group $(p=0.002)$. Nakashima et $\mathrm{al}^{(7)}$ studied subgroups of patients who would benefit from ECPR, with a focus on transition of cardiac rhythm during OHCA. The study showed that there was a more favourable outcome in sustained VF/VT $(19.7 \%)$ compared to rhythm change $(3.3 \%)(p<0.001)$, with the rate of favourable outcomes increasing 5.5-fold for sustained VF/VT with ECPR. ${ }^{(7)}$ Their study also showed that the following factors could have a favourable outcome: age (OR 0.94); witnessed arrest (OR 1.46); bystander CPR (OR 2.08); time from OHCA to ECPR initiation (OR 0.99); and acute coronary syndrome (OR 0.51). Both of these studies incorporated the use of therapeutic hypothermia and intra-aortic balloon pump together with ECPR. ${ }^{(6,7)}$

Studies have also shown that ECPR is similarly beneficial for in-hospital cardiac arrest (IHCA) patients. Chen et al concluded that ECPR has both short- and long-term benefits for IHCA patients; propensity analysis comparing ECPR to CCPR showed a significant difference favouring ECPR, in terms of survival to discharge, 30-day survival rate and one-year survival rate. ${ }^{8}$ Shin et al studied IHCA patients in a single centre who received CPR for more than ten minutes, using survival to discharge with minimal neurological deficit as the endpoint. ${ }^{(9)}$ Their study showed significantly better survival rates in the ECPR group as compared to the CCPR group, in terms of survival to discharge (OR 0.19) and six-month survival (hazard ratio 0.48). A subgroup analysis of IHCA due to cardiac causes showed better results in the ECPR group. Although the OR and hazard ratio are small, it is nonetheless encouraging that ECPR may also prove to be beneficial for IHCA patients who received more than ten minutes of CPR and cardiac-related arrest patients. ${ }^{(9)}$ In a subsequent two-year follow-up study in 2013, Shin et al showed a four-fold increase in survival with minimal neurological deficit in the ECPR group, and found that age $<65$ years and CPR duration $<35$ minutes were independent predictors of survival with minimal neurological deficit. ${ }^{(10)}$

A study by Wang et al showed that ECPR was beneficial for both IHCA and OHCA patients. ${ }^{(11)}$ The CHEER (mechanical CPR, Hypothermia, ECMO and Early Reperfusion) trial, ${ }^{(12)}$ conducted at The Alfred Hospital, Australia, utilised therapeutic hypothermia and early reperfusion in addition to ECPR. Comprising 26 patients (mean age 52 years) - 15 IHCA and 11 OHCA - the study reported that $54 \%$ of the patients survived to hospital discharge with CPC 1. The Alfred Hospital has since adopted this regime as a standard of care. ${ }^{(12)}$

\section{PUTTING EVIDENCE INTO PRACTICE}

ECPR is a potentially resource-intensive procedure in terms of manpower and other medical resources. In an already overburdened medical system, implementing ECPR can lead to wasted resources, which could otherwise be utilised for other patients. The American Heart Association recognised this important caveat and stated in its latest guidelines that the goal of ECPR was to support patients in refractory cardiac arrest while reversible causes are being identified and treated; ${ }^{(13)}$ ELSO's 2013 recommendation ${ }^{(13)}$ was in agreement with this guideline. In addition, ELSO recommended that it is medically futile to proceed with ECPR if CPR has been unsuccessful for 30 minutes. ${ }^{(14)}$ This recommendation is in keeping with the aforementioned evidence. However, if effective CPR is performed with adequate circulatory volume, providing sufficient perfusion and metabolic support during compressions, ECPR may still be considered. Surrogate markers such as arterial blood gas may be used as a measure of effectiveness of CPR, while the presence of severe metabolic acidosis may indicate poor perfusion during CPR.

To ensure best outcomes, adherence to the suggested guiding principles and better use of limited medical resources, it is extremely important to have strict patient selection criteria. Many studies have used rigorous inclusion and exclusion criteria with wide cross-study variations. More consistent criteria include patient age of 18-75 years, suspected cardiac origin of cardiac arrest and continuous CPR $\leq 10$ minutes from recognition of cardiac arrest. Other criteria that should be taken into consideration for patient selection include: initial or persistent VF/VT rhythm, performance of bystander CPR and witnessed cardiac arrest. Exclusion criteria for ECMO include: patients with a do-not-resuscitate order, end-organ dysfunction and/or suspicion of aortic dissection.

It is also without doubt that performing ECPR in a centre with a specialised ECMO service will more likely yield better results. Luft et al ${ }^{(15)}$ showed that mortality rates for high-risk surgeries were $25 \%-41 \%$ lower in centres that performed over 200 such surgeries. Barbaro et $\mathrm{al}^{(16)}$ also found a strong association between institutions with high ECMO volume and lower mortality in adults and neonates. These two examples point to the importance of having the appropriate expertise to perform the procedure, having ECMO centres with high caseloads and selecting the right patients to benefit from the procedure.

\section{CONCLUSION}

ECPR is only one component of the post-cardiac arrest regimen (which includes therapeutic hypothermia, early reperfusion, treatment of root cause, tight glycaemic control and low tidal volume ventilation) and functions as a bridge to definitive therapy. As the CHEER trial and studies on therapeutic hypothermia have aptly demonstrated, therapeutic hypothermia and early reperfusion can give excellent patient outcomes. It is thus important to complement ECPR with good post-cardiac arrest care. ECPR is still an evolving field; as more studies are conducted and clinical experience improves, there is hope that patients who would otherwise die will be given a second chance at life. 


\section{REFERENCES}

1. Brown K, Dalton H. Extracorporeal Cardiopulmonary Resuscitation: ECPR. In: Annich G, Lynch W, MacLaren G, Wilson J, Bartlett R, eds. ECMO Extracorporeal Cardiopulmonary Support in Critical Care. 4th ed. Ann Arbor, MI: Extracorporeal Life Support Organization, 2012: 331-40.

2. Wollenek G, Honarwar N, Golej J, Marx M. Cold water submersion and cardiac arrest in treatment of severe hypothermia with cardiopulmonary bypass. Resuscitation 2002; 52:255-63.

3. Chan T, Thiagarajan RR, Frank D, Bratton SL. Survival after extracorporeal cardiopulmonary resuscitation in infants and children with heart disease. J Thorac Cardiovasc Surg 2008; 136:984-92.

4. Extracorporeal Life Support Organization. International ECLS Registry 2011. Ann Arbor, MI: 2011.

5. Cardarelli MG, Young AJ, Griffith B. Use of extracorporeal membrane oxygenation for adults in cardiac arrest (E-CPR): a meta-analysis of observational studies. ASAIO J 2009; 55:581-6.

6. Sakamoto T, Morimura N, Nagao K, et al; SAVE-J Study Group. Extracorporeal cardiopulmonary resuscitation versus conventional cardiopulmonary resuscitation in adults with out-of-hospital cardiac arrest: a prospective observational study. Resuscitation 2014; 85:762-8.

7. Nakashima T, Tahara Y, Yasuda S, et al. Abstract 11816: Sustained ventricular fibrillation during CPR as the strongest predictor for the favorable neurological outcomes in out-of-hospital cardiac arrest patients: the multicenter prospective study of SAVE-J. Circulation 2015; 132(Suppl 3).

8. Chen YS, Lin JW, Yu HY, et al. Cardiopulmonary resuscitation with assisted extracorporeal life-support versus conventional cardiopulmonary resuscitation in adults with in-hospital cardiac arrest: an observational study and propensity analysis. Lancet 2008; 372:554-61.

9. Shin TG, Choi JH, Jo IJ, et al. Extracorporeal cardiopulmonary resuscitation in patients with inhospital cardiac arrest: a comparison with conventional cardiopulmonary resuscitation. Crit Care Med 2011; 39:1-7.

10. Shin TG, Jo IJ, Sim MS, et al. Two-year survival and neurological outcome of in-hospital cardiac arrest patients rescued by extracorporeal cardiopulmonary resuscitation. Int J Cardiol 2013; 168:3424-30.

11. Wang CH, Chou NK, Becker LB, et al. Improved outcome of extracorporeal cardiopulmonary resuscitation for out-of-hospital cardiac arrest--a comparison with that for extracorporeal rescue for in-hospital cardiac arrest. Resuscitation 2014; 85:1219-24.

12. Stub D, Bernard S, Pellegrino $V$, et al. Refractory cardiac arrest treated with mechanical CPR, hypothermia, ECMO and early reperfusion (the CHEER trial). Resuscitation 2015; 86:88-94.

13. de Caen AR, Berg MD, Chameides L, et al. Part 12: Pediatric Advanced Life Support: 2015 American Heart Association Guidelines Update for Cardiopulmonary Resuscitation and Emergency Cardiovascular Care. Circulation 2015; 132(18 Suppl 2):S526-42.

14. Extracorporeal Life Support Organization (ELSO) Patient Specific Supplements to the ELSO General Guidelines. April 2009. Available at: http://square.umin. ac.jp/jrcm/pdf/ecmo/ecmotext12.pdf. Accessed July 23, 2016.

15. Luft HS, Bunker JP, Enthoven AC. Should operations be regionalized? The empirical relation between surgical volume and mortality. N Engl J Med 1979; 301:1364-9.

16. Barbaro RP, Odetola FO, Kidwell KM, et al. Association of hospital-level volume of extracorporeal membrane oxygenation cases and mortality. Analysis of the extracorporeal life support organization registry. Am J Respir Crit Care Med 2015; 191:894-901. 